\title{
Conoscerlo per riconoscerlo: morbo di Addison con sindrome poliendocrina autoimmune di
} Tipo 2

\author{
Marco Lombardi ${ }^{1}$, Stefano Michelassi ${ }^{1}$, Corrado Betterle ${ }^{2}$ \\ ${ }^{1}$ S.C di Nefrologia e Dialisi, Ospedale SM Annunziata e del Mugello, Azienda Sanitaria di Firenze, Firenze \\ ${ }^{2}$ Dipartimento di Medicina, U.O.C. di Endocrinologia, Azienda Ospedaliera-Università Padova, Padova
}

In collaborazione con AIPAd (Associazione Italiana Pazienti Addison)

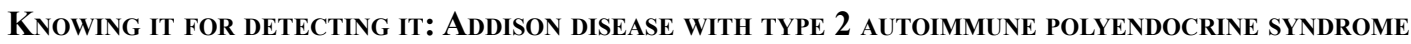

Abstract. Here we describe a patient who developed autoimmune hypoadrenalism many years after developing Graves' disease. This combination depicts an autoimmune polyendocrine syndrome type-2 (APS-2).

APS-2 is an autoimmune syndrome involving endocrine (adrenal glands + thyroid, and/or type 1 diabetes mellitus) and non-endocrine autoimmune disorders. APS-2 has a genetic background showing an association with the class II major histocompatibility complex. APS-2 is an uncommon disease (1:8,000 inhabitants) with higher prevalence in females and mean onset age of 35 years.

Despite the fact that hypoadrenalism is characterized by specific symptoms and signs (asthenia, fatigue, orthostatic hypotension, weight loss, nausea, arthralgia, anorexia, skin hyperpigmentation) it is not easy to diagnose at early stages. In late referral patients clinical symptoms can worsen, due to stress events, until a possibly fatal addisonian crisis.

Hyponatremia, hyperkalemia, increased levels of ACTH, renin, and low levels of cortisol, hepatic failure as well as clinical signs and symptoms of dehydration are late laboratory findings.

The natural history of the disease develops in different, progressive phases: (i) presence of adrenal cortex autoantibodies many years before the first clinical manifestations, (ii) initial increased levels of plasmatic renin and low levels of aldosterone, (iii) followed by a reduced response of cortisol to the intravenous ACTH stimulation, (iv) and finally increased levels of plasmatic ACTH and low levels of cortisol and clinical manifestations of adrenal insufficiency. The therapy of APS-2 is mainly focused on hormone therapy of the affected glands.
\end{abstract}

Key words: Addison's disease, Autoimmune polyendocrine syndrome type-2

Conflict of interest: None.

Ricevuto: 2 Dicembre, 2012; Accettato: 1 Gennaio 2013

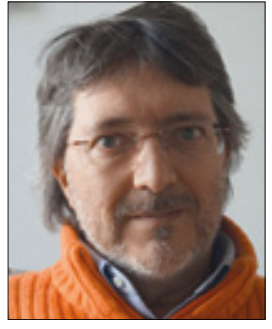

Marco Lombardi

\section{Il caso clinico}

Si tratta di un paziente maschio di 57 anni di razza caucasica, con familiarità per diabete mellito tipo 2, cardiopatia ischemica, morti improvvise e atopia. Pregresso modesto fumatore ( 3 sigarette al giorno), con lunga storia di atopia a pollini, polveri e alimenti. In età pediatrica il paziente contrasse, oltre alle comuni malattie infantili, anche la poliomelite, della quale sono residuati modesti esiti (lieve deficit degli adduttori interni della coscia $\mathrm{dx}$ ).

All'età di 25 anni venne diagnosticata un'ernia jatale da scivolamento. A 33 anni fu sottoposto a intervento neurochirur- gico per ematoma extradurale temporale $\mathrm{dx}$ post-traumatico $\mathrm{e}$ a 50 a polipectomia endoscopica del colon.

All'età di 43 anni venne diagnosticato il morbo di Graves, trattato prima con metimazolo e beta-bloccanti e successivamente con ${ }^{131} \mathrm{I}$ e boli e.v. di steroidi per l'oftalmopatia, con successivo ipotiroidismo, trattato con levo-tiroxina.

All'età di 45 anni fu riscontrata un'ipertensione arteriosa diastolica, trattata con beta-bloccanti e diuretici.

A 53 anni venne colpito da polmonite di probabile origine virale. $\mathrm{Da}$, allora, il paziente cominciò ad accusare artromialgie diffuse e soprattutto uno stato di astenia e depressione a così lenta progressione da essere interpretata all'inizio come stato protratto di astenia post-infettiva. Tuttavia, tale situazione si protrasse nei mesi successivi e venne interpretata dapprima come parte di un "fisiologico" invecchiamento 
TABELLA I - PRINCIPALI ESAMI EMATOCHIMICI DAL 2004 AL GIUGNO 2012

\begin{tabular}{lccccccccccc}
\hline & $\mathbf{2 0 0 4}$ & $\mathbf{2 0 0 5}$ & $\mathbf{2 0 0 6}$ & $\mathbf{2 0 0 7}$ & $\mathbf{2 0 0 9}$ & $\mathbf{2 0 1 0}$ & $\mathbf{2 0 1 1}$ & $\mathbf{4 / 6 / 2 0 1 2}$ & $\mathbf{1 8 / 6 / 2 0 1 2}$ & V.N. \\
\hline Creatinina & 0.82 & 0.77 & 0.92 & 0.9 & 0.75 & 0.93 & 0.87 & 0.96 & 1.03 & $0.7-1.2 \mathrm{mg} / \mathrm{dL}$ \\
$\mathrm{Na}$ & 141 & 141 & 143 & 141 & 138 & 134 & 138 & 135 & 133 & $136-145 \mathrm{mEq} / \mathrm{L}$ \\
$\mathrm{K}$ & 4.5 & 4.8 & 4.7 & 4.3 & 4.4 & 4.8 & 4.6 & 5.1 & 5.3 & $3.5-5.1 \mathrm{mEq} / \mathrm{L}$ \\
Ac. urico & 5.6 & 6.0 & 5.9 & 5.2 & 4.9 & 5.6 & - & 7.1 & 7.8 & $3.4-7.0 \mathrm{mg} / \mathrm{dL}$ \\
$\mathrm{Hb}$ & 14.5 & 14.7 & 14.5 & 15.9 & 13.7 & 15.6 & 15.3 & 16.1 & 17.3 & $13.2-17 \mathrm{~g} / \mathrm{dL}$ \\
AST/ALT & $23 / 25$ & $22 / 21$ & $18 / 19$ & $18 / 18$ & $21 / 22$ & $26 / 32$ & $23 / 24$ & $36 / 55$ & $44 / 70$ & $<37 \mathrm{e}<41 \mathrm{UI} / \mathrm{L}$ \\
$\gamma$ GT & 40 & 38 & 30 & 34 & 27 & 32 & 29 & 115 & 144 & $7-50 \mathrm{U} / \mathrm{L}$ \\
PCR & 0.13 & 0.34 & 0.15 & 1.38 & 0.64 & 0.13 & 0.33 & 0.73 & 0.93 & $<0.5 \mathrm{mg} / \mathrm{dL}$ \\
s-TSH & 0.53 & 0.82 & 2.17 & 4.6 & 1.65 & 1.39 & 1.99 & 0.93 & - & $0.35-5.50 \mathrm{mU} / \mathrm{L}$ \\
\hline
\end{tabular}

anagrafico e successivamente come conseguente a stress fisici o emotivi intercorrenti.

All'età di 56 anni questo stato di malessere generale diventò sempre più accentuato, con eccessiva sonnolenza e difficoltà a svolgere anche le comuni attività, e comparve anche un progressivo bisogno di assumere cibi salati. Negli ultimi mesi si aggiunsero turbe gastrointestinali (nausea, malessere gastrico, difficoltà digestive, continua sensazione di fame pur con difficoltà digestive) e cefalea subcontinua ma accentuata specialmente dal passaggio dal clino- all'ortostatismo e iperosmia.

Per la concomitante presenza di infezioni batteriche (Streptococco beta-emolitico) e virali nell'ambito del proprio nucleo familiare, tale sintomatologia venne attribuita a un possibile processo infettivo, ipotesi suffragata dal lieve aumento di enzimi epatici e dei marker di flogosi nel corso di esami effettuati a inizio Giugno 2012 all'età di 57 anni.

Tra gli esami eseguiti in tale periodo venne documentata anche una progressiva diminuzione della sodiemia con aumento di potassiemia e con emoglobina ancora nel range dei valori normali (Tab. I). Negli ultimi giorni prima del ricovero, un controllo pressorio rilevò una modesta ipertensione arteriosa $(140 / 95 \mathrm{mmHg})$ e il paziente fu invitato a ridurre l'apporto di sale e a potenziare la terapia antiipertensiva.

Tali misure furono seguite da un ulteriore peggioramento delle condizioni cliniche generali con profonda astenia e comparsa di grave ipotensione arteriosa $(80 / 40 \mathrm{mmHg}$ in clinostatismo), per cui vennero sospesi i farmaci antiipertensivi e il paziente chiese il ricovero. Nel frattempo si era manifestata anche la presenza di un colorito particolarmente "abbronzato" del paziente, con accentuazione della pigmentazione a livello delle pieghe cutanee palmari, sintomi ai quali non venne data una particolare interpretazione, vista la stagione estiva.

Ricoverato in Day Hospital infettivologico nei primi giorni del Luglio 2012, gli esami mostrarono un'ulteriore alterazione degli enzimi epatici e l'ecografia addominale rilevò una modesta epatomegalia con componente steatosica. Tuttavia, gli esami evidenziarono anche progressive iposodiemia e iperpotassiemia, la TC addome mostrò ipotrofia di ambedue le ghiandole surrenali e la sintomatologia migliorò rapidamente dopo l'infusione endovenosa di $1500 \mathrm{cc}$ di soluzione fisiologica. L'orientamento diagnostico si spostò quindi da un'epatopatia infettivo-steatosica a un iposurrenalismo (morbo di Addison)
TABELLA II - ESAMI A CONFERMA DI MORBO DI ADDISON SU BASE AUTOIMMUNE

\begin{tabular}{lll}
\hline Test & Valore riscontrato & Range di normalità \\
\hline ACTH plasmatico & 790.7 & {$[7-63 \mathrm{pg} / \mathrm{mL}]$} \\
Cortisolo urinario & 12.4 & {$[36-137 \mathrm{ug} / \mathrm{die}]$} \\
Aldosterone urinario & 0.01 & {$[7.8-83.4 \mathrm{umol} /$ die $]$} \\
Renina plasm. orto. & n.p. & {$[3-33 \mathrm{pg} / \mathrm{mL}]$} \\
AA anti-surrene & Fortemente positivi & Neg. \\
Altri AA & Tutti negativi & Neg. \\
\hline
\end{tabular}

n.p $=$ non pervenuta $\quad A A=$ autoanticorpi

confermato dagli esami endocrinologici specifici a eziologia autoimmune, vista la positività degli anticorpi anti-surrene (Tab. II) e data la presenza anamnestica del morbo di Graves, verso una sindrome poliendocrina autoimmune di tipo 2 (SPA-2).

Venne prescritta una terapia con cortone acetato $25 \mathrm{mg}, 1 \mathrm{cp} \mathrm{a}$ ore 8.00 e a ore 14.00 , che produsse un rapido miglioramento delle condizioni generali, con il ripristino di una pressione arteriosa $100-105 / 70-80 \mathrm{mmHg}$ in ortostatismo.

Lo specialista endocrinologo successivamente aggiustò la terapia prescrivendo idrocortisone $(20 \mathrm{mg}$ a ore $8.00,15 \mathrm{mg}$ a ore 14.00 e $5 \mathrm{mg}$ a ore 19.00), associato a fludrocortisone (0.1 mg al mattino) per via orale.

\section{Discussione}

Il primo caso individuato di SPA è ascrivibile proprio allo scopritore del morbo di Addison, ovvero a Thomas Addison, che nel 1855 descrisse un paziente affetto da iposurrenalismo "idiopatico" e vitiligo (1). Successivamente sempre T. Addison descrisse in un altro paziente, anche l'anemia perniciosa, definita sindrome di Addison-Biermer.

La prima associazione tra morbo di Addison e tiroidite cronica (peraltro senza disfunzione tiroidea clinicamente evidente) fu descritta in due pazienti da Schmidt nel 1926 (2) da cui la denominazione di sindrome di Schmidt.

Nel 1931, Rowntree riportò il primo caso di morbo di Addison associato a ipertiroidismo e a diabete mellito (3) e nel 1932 
TABELLA III - CLASSIFICAZIONE DELLE SPA SECONDO NEUFELD 1980 (6)

SPA-1 Candidiasi cronica, ipoparatiroidismo cronico, Addison (almeno due presenti)

SPA-2 Addison (sempre presente), tiroidite autoimmune e/o diabete mellito tipo $1^{*}$

SPA-3 Tireopatia autoimmune (tiroidite cronica o M. di Graves) associata a un'altra malattia autoimmune che non sia l'Addison:

A) Diabete mellito di tipo 1

B) Gastrite autoimmune con o senza anemia perniciosa

C) Vitiligine o alopecia o miastenia gravis

SPA-4 Combinazioni non comprese nei tre gruppi precedenti

(* autoimmune)

Gowen descrisse il primo caso di morbo di Addison associato a ipotiroidismo e a diabete mellito (4). Il paziente morì in chetoacidosi e l'analisi post-mortem dimostrò in alcune insule pancreatiche un'infiltrazione linfocitaria simile a quella presente nella corticale surrenalica e nella tiroide e ciò fece ipotizzare una causa comune delle tre malattie.

Nel 1957 furono rivelati, in pazienti con morbo di Addison idiopatico (ovvero non tubercolare), autoanticorpi circolanti contro estratti di tessuto surrenalico che portarono a ipotizzare una causa autoimmune per tale malattia (5).

Nel 1980 fu pubblicata la prima classificazione clinica delle "autoimmune polyendocrine syndromes" di cui l'acronimo in inglese è APS, ma definite sindromi poliendocrine autoimmuni (SPA) in Italiano (Tab. III) (6).

A oggi si ritiene che le SPA, come molte altre malattie autoimmuni, per manifestarsi necessitino di una predisposizione genetica che permetta a ipotetici fattori ambientali di stimolare il sistema immunitario a perdere la tolleranza immunologica verso antigeni propri dell'organismo e ad aggredirli come fossero antigeni estranei (7).

La SPA-1 è una malattia monogenica autosomica recessiva molto rara che compare e si sviluppa di solito in età pediatrica, dovuta a mutazioni del gene (AIRE, Auto-Immune REgulator) posto sul cromosoma 21 , che codifica per un fattore di trascrizione espresso nel timo.

In questa malattia il morbo di Addison si manifesta in età molto giovane (tra gli 8 e i 14 anni di età) preceduto da una candidiasi cronica muco-cutanea e dall'ipoparatiroidismo. Molte altre malattie autoimmuni e non autoimmuni possono manifestarsi con frequenza elevata in aggiunta a questa triade classica durante tutto l'arco della vita e la sindrome può presentare anche una distrofia ectodermica, per cui tale sindrome viene anche denominata nella letteratura internazionale con l'acronimo di APECED che sta per Autoimmune-Poly-Endocrinopathy-Candidiasis-Ectodermal-Dystrophy (8).

La SPA-2 è invece una malattia poligenica associata al complesso maggiore di istocompatibilità MCH II localizzato sul cromosoma 6 , in particolare agli aplotipi HLA-DR3 (DQB*0201) e DR4 (DQB1*0302), ma anche a geni nonHLA (MICA5.1, PTPN22, CTLA4, VNTR, ecc.) (7, 9, 10). L'ereditarietà della SPA-2 sembra autosomica dominante con penetranza incompleta; i parenti di primo grado dei sogget- ti affetti hanno un aumentato rischio di malattie autoimmuni (specialmente tiroidee) (9) e circa la metà dei pazienti ha una storia familiare di patologie autoimmuni (11).

La SPA-2, o sindrome di Schmidt, è più frequente della SPA1 , compare di solito nella donna adulta (9) ed è caratterizzata dall'associazione di morbo di Addison, tireopatia autoimmune (tiroidite cronica o morbo di Graves) e/o DM-1.

Un recente studio italiano fatto su 322 pazienti con SPA-2 ha dimostrato che l'età media di comparsa del morbo di Addison è di 34.6 anni, del DM-1 di 28.5 anni e delle malattie autoimmuni della tiroide di 35.8 anni. Il rapporto tra femmine e maschi è di 2.3/1 e quello tra adulti e bambini di 16/1 (Betterle osservazione personale). La frequenza della combinazione con cui le 3 malattie principali si associano è riassunta nella Tabella IV. Dall'analisi di tali dati si dimostra che, nella stragrande maggioranza dei casi (90.4\%), sono presenti associazioni varie di due malattie: morbo di Addison con tireopatie autoimmuni (come nel caso del nostro paziente) o morbo di Addison con il DM-1, mentre l'associazione delle tre componenti è osservabile solo nel $9.6 \%$ dei casi. La tiroidite cronica si manifesta prima del morbo di Addison nel 49\% dei casi, nel $28 \%$ dei casi contemporaneamente e nel $23 \%$ dei casi successivamente. Il morbo di Graves si diagnostica prima del morbo di Addison nel 71\% dei casi (come avvenuto nel caso del nostro paziente), contemporaneamente nel $2 \%$ dei casi e successivamente nel $27 \%$ dei casi. Il DM-1 si osserva prima della diagnosi di morbo di Addison nel 71\% dei casi, alla diagnosi nel $14 \%$ dei casi e successivamente nel 16\% dei casi (Betterle osservazione personale).

Nella SPA-2 possono essere presenti altre malattie autoimmuni minori quali malattia celiaca, gastrite cronica atrofica con e senza anemia perniciosa, vitiligine, alopecia, ipogonadismo ipergonadotropico, ipofisite, miastenia grave, sindrome dell'uomo rigido (stiff-man syndrome), epatite autoimmune, cirrosi biliare primitiva, artrite sieronegativa, sindrome di Sjogren, morbo di Parkinson e porpora trombotica trombocitopenica (9). Tuttavia, queste si manifestano con minore frequenza rispetto alla SPA-1.

Per quanto riguarda la presentazione clinica del morbo di Ad-

TABELLA IV - FREQUENZA DELLE TRE PRINCIPALI MALATTIE SU 322 PAZIENTI CON SPA-2 (Betterle osservazione personale)

\begin{tabular}{lcc}
\hline Combinazioni & Numero dei casi & Frequenza (\%) \\
\hline Morbo di Addison + Tiroidite cronica & $236 / 322$ & 73.4 \\
& & 10.8 \\
Morbo di Addison + morbo di Graves & $35 / 322$ & \\
& & 7.7 \\
$\begin{array}{l}\text { Morbo di Addison + Tiroidite cronica } \\
\text { + Diabete Mellito di tipo 1 }\end{array}$ & $25 / 322$ & 6.2 \\
$\begin{array}{l}\text { Morbo di Addison + Diabete Mellito } \\
\text { di tipo 1 }\end{array}$ & $20 / 322$ & 1.9 \\
$\begin{array}{l}\text { Morbo di Addison + morbo di Graves } \\
\text { + Diabete Mellito di tipo 1 }\end{array}$ & $6 / 322$ & \\
\hline
\end{tabular}


dison è frequentemente caratterizzata da sintomi che si appalesano inizialmente in modo insidioso o fluttuante, che possono avere una progressione molto lenta e che possono essere comuni a molte altre patologie (10): facile stancabilità, malessere generale, apatia, anoressia e perdita di peso. La debolezza e l'affaticamento sono generalizzati, progressivi e peggiorano con l'attività fisica. La perdita di peso (descritti casi anche fino a $15 \mathrm{Kg}$ ) è ascrivibile sia all'anoressia che alla disidratazione. Tali sintomi sono progressivi e si associano spesso al bisogno di mangiare sale. Nei casi che rimangono non diagnosticati si può arrivare alla "crisi" addisoniana o surrenalica, emergenza potenzialmente fatale, spesso precipitata da uno stress psicofisico (infezione, malattia intercorrente, trauma, intervento chirurgico, anestesia, ecc.) (7).

Il corteo sintomatologico generale è accompagnato da sintomi gastrointestinali, anch'essi poco specifici come nausea, vomito, dolori addominali, diarrea o stipsi; il vomito e la diarrea possono essere sintomi precoci di un'incipiente crisi surrenalica. La manifestazione cardiovascolare principale è l'ipotensione arteriosa, più marcata in ortostatismo, e la comparsa di un evento sincopale nel paziente addisoniano può essere la conseguenza di una grave ipotensione o di un'ipoglicemia (dovuta a fattori multipli quali il digiuno prolungato e la gluconeogenesi deficitaria e alla carenza del cortisolo, che è il maggiore ormone controinsulare). Più raramente possono essere presenti anche alterazioni della sfera sessuale (p. es., calo della libido, amenorrea, riduzione dei peli ascellari o pubici) e sintomi neuropsicologici (confusione, depressione e psicosi) (7).

In tutti i casi di morbo di Addison è osservabile la caratteristica iperpigmentazione cutanea ("morbo bronzino") più o meno evidente nelle pieghe del palmo delle mani, nelle aree già normalmente iperpigmentate (capezzoli, nevi) e nelle cicatrici recenti, ma presente anche sulle mucose orali e gengivali. L'iperpigmentazione è dovuta a un'aumentata sintesi di melanina per iperincrezione di proopiomelanocortina (polipeptide sintetizzato nell'adenoipofisi sotto stimolo del CRH e precursore, oltre che dell'ACTH, di altri peptidi fra cui MSH). Tale segno è invece assente nelle forme di iposurrenalismo di origine centrale dove vi è un deficit della secrezione di ACTH. Gli esami di laboratorio evidenziano soprattutto iposodiemia (con ipoosmolarità plasmatica) e iperpotassiemia ma anche tendenza all'ipocloremia e all'ipercalcemia, lieve incremento degli indici di epatolisi e, talvolta, ipoglicemia. Tuttavia, tali alterazioni sono relativamente tardive (10).

La diagnosi richiede la dimostrazione di elevati livelli plasmatici di ACTH e ridotti livelli (urinari, plasmatici o salivari) di cortisolo. È ridotta anche la produzione di aldosterone, a fronte di un'aumentata attività reninica plasmatica (7).

Alla diagnosi nel siero dei pazienti con morbo di Addison autoimmune sono dimostrabili anticorpi anti-corteccia surrenalica (ACA) e/o anti-21-idrossilasi (21-OHAbs), presenti in oltre il $90 \%$ dei casi (7). Questi autoanticorpi possono essere rilevati anche mesi o anni prima della manifestazione clinica della malattia (13).

Nei pazienti con la forma autoimmune (cioè con morbo di Addison positivo per ACA e/o 21-OHAbs), le indagini strumentali quali la TAC o la RMN hanno sempre mostrato la presenza di ghiandole surrenali di volume normale o piccolo, tanto che ora, in caso di positività, non è più consigliato eseguire l'indagine morfologica dei surreni. La TAC o la RMN dei surreni associate ad altre indagini specifiche sono invece necessarie nei pazienti con morbo di Addison negativo per ACA e/o 21-OHAbs, in quanto possono permettere di sospettare altre forme di morbo di Addison quali quelle post-TBC, infettive, vascolari, neoplastiche, genetiche, ecc. (14).

Nei pazienti con morbo di Addison autoimmune, come già detto, possono essere presenti altre patologie autoimmuni clinicamente evidenti marcate dalla presenza dei rispettivi autoanticorpi $(7,9)$. Tuttavia autoanticorpi organo-specifici (anti-tiroidei, anti-stomaco, anti-fattore intrinseco, anti-insula pancreatica, anti-decarbossilasi dell'acido glutammico, antitransglutaminasi, anti-cellule steroido-secernenti) possono essere presenti in assenza di tali patologie, ma la loro presenza richiede l'esecuzione di indagini specifiche (p. es., TSH e FT4 per gli anticorpi anti-tiroidei, gastrinemia ed emocromo per gli anticorpi anti-stomaco, glicemia e HbAlc per gli anticorpi anti-pancreas endocrino, gastroduodenoscopia per gli anticorpi anti-transglutaminasi, FSH, LH, estradiolo per gli anticorpi anti-cellule steroido-secernenti, ecc.) $(12,16)$. Tali test permettono di diagnosticare forme o subcliniche o potenziali di APS-2, ma a rischio di sviluppare nel tempo la sindrome completa.

Il trattamento del morbo di Addison è imperniato sulla terapia sostitutiva ormonale. La carenza di glucocorticoidi richiede la sostituzione con idrocortisone (20-30 mg/die per os) o con cortone acetato (25-37.5 mg/die) in due o tre somministrazioni (la dose maggiore al risveglio, la seconda dose dopo 6 ore circa e la terza verso le 18.00-19.00), al fine di mimare il ritmo circadiano del cortisolo e di controllare i sintomi clinici della malattia.

In caso di basso dosaggio, il paziente potrà avere persistenza delle manifestazioni cliniche del morbo di Addison o comunque un non completo benessere, mentre, in caso di iperdosaggio, i pazienti svilupperanno aumento di peso corporeo, ipertensione, osteoporosi, gastropatia e così via. Il dosaggio del cortisolo urinario, plasmatico o salivare è l'esame fondamentale per monitorare l'appropriatezza della dose assunta (7).

Il deficit di mineralcorticoidi richiede la somministrazione di fludrocortisone, generalmente assunto alla dose di 0.05-0.15 $\mathrm{mg} /$ die in dose unica al mattino al risveglio. L'appropriatezza di questa sostituzione ormonale è valutata con parametri clinici (pressione arteriosa, peso corporeo, eventuali segni di ritenzione idrosalina) e biochimici (sodiemia, potassiemia, renina plasmatica in ortostatismo) (7). Nei casi di interessamento contemporaneo surrenalico e tiroideo deve essere trattato prima l'iposurrenalismo e poi l'ipotiroidismo, perché la tiroxina, aumentando il metabolismo epatico dei corticosteroidi, può far precipitare una crisi surrenalica, se il paziente non è ancora trattato con dosi adeguate di steroidi (11).

La mortalità nei pazienti con insufficienza surrenalica primitiva nella popolazione nord-europea è approssimativamente doppia rispetto alla popolazione di controllo, principalmente per la crisi addisoniana o per complicanze endocrine (perlopiù dovute al diabete) o per problemi cardiovascolari (15). Dati preliminari ottenuti nella popolazione italiana con morbo di Addison non sembrano confermare tale elevata mortalità 
(Betterle et al., dati personali).

Ogni paziente addisoniano deve essere reso consapevole della necessità di assumere $i$ farmaci sostitutivi giornalmente per tutta la vita e che i dosaggi di tali farmaci devono essere aumentati in condizioni di stress o malattia intercorrente. Infatti, la terapia orale va adeguata o sostituita con terapia parenterale in caso di stress fisico o emotivo, eventi febbrili e gastrointestinali, traumi o interventi chirurgici maggiori o minori.

\section{Conclusione}

La SPA-2 è una sindrome autoimmune in cui alcune ghiandole endocrine, quali corteccia surrenalica, tiroide e isole pancreatiche, possono essere colpite e distrutte dal sistema immune. $\mathrm{Nel}$ caso particolare dei surreni, la loro distruzione porta allo sviluppo del morbo di Addison, malattia ancora oggi a esito fatale, se non diagnosticata tempestivamente. Il punto cruciale di questa malattia sta proprio nella difficoltà di riconoscerla. Da qui, la necessità di una maggiore consapevolezza di essa (e delle sindromi poliendocrine in generale) tra i medici. Infatti, una corretta interpretazione dei sintomi e dei segni in presenza di iposodiemia e di iperpotassiemia potrà permettere di considerare nella diagnosi differenziale l'insufficienza corticosurrenalica, diagnosi che potrà prevenire la comparsa di una crisi surrenalica, evento potenzialmente fatale. Il paziente potrà allora essere inviato allo specialista endocrinologo, a cui competeranno la conferma della diagnosi mediante esami specifici come il dosaggio dell'ACTH e del cortisolo plasmatici, del cortisolo urinario, dell'aldosterone urinario e dell'attività plasmatica della renina e l'identificazione della patogenesi con la ricerca degli ACA e/o dei 21-OHAbs ed eventuali ulteriori indagini.

A questo proposito è utile ricordare che gli ACA e/o i 21 -OHAbs sono presenti in oltre il $90 \%$ dei pazienti con morbo di Addison al momento della diagnosi ma possono essere dimostrati anche molti anni prima della comparsa della malattia (13).

Dato che circa 1' $80 \%$ dei pazienti con morbo di Addison ha o è destinato a sviluppare nel tempo altre patologie autoimmuni, in tutti i pazienti con iposurrenalismo autoimmune è raccomandabile la ricerca di altre patologie autoimmuni cliniche mediante determinazione di TPO esami specifici di laboratorio e, in particolare, di patologie tiroidee e diabete mellito.

In caso di assenza di tali patologie cliniche, lo screening autoanticorpale potrà essere utile mediante TPO-abs e TgAbs per identificare una patologia subclinica o potenziale della tiroide mediante ricerca di anticorpi anti-cellule parietali gastriche e anti fattore intrinseco per identificare una patologia subclinica o potenziale pancreatica endocrina, mediante ricerca di anticorpi anti-cellule steroido-secernenti per identificare per identificare una patologia subclinica o potenziale autoimmune delle gonadi e mediante ricerca di anticorpi anti-transglutaminasi per identificare una patologia subclinica o potenziale celiaca.

I casi positivi senza alterazione dei rispettivi organi bersaglio dovranno essere comunque seguiti con periodici test specifici per l'individuazione dello sviluppo di un'eventuale patologia subclinica o clinica. Nei pazienti inizialmente negativi, tale screening dovrà essere ripetuto periodicamente ogni 2-3 anni $(16,17)$.

Inoltre, dato che le patologie autoimmuni e il morbo di Addison hanno una predisposizione genetica, tali patologie autoimmuni cliniche o latenti dovranno essere ricercate nei parenti di primo grado dei pazienti in cui sia stata fatta diagnosi di SPA. Infine, parafrasando il motto divenuto famoso a proposito dell'AIDS ("se lo conosci lo eviti"), per il morbo di Addison possiamo certamente dire "se lo conosci lo riconosci".

\section{Riassunto}

Viene presentato un caso clinico di iposurrenalismo da morbo di Addison primitivo sviluppatosi dopo alcuni anni dalla comparsa di un morbo di Graves. Tale combinazione rappresenta una poliendocrinopatia autoimmune di tipo 2 (SPA-2). La SPA-2 è un processo autoimmune che coinvolge più tessuti endocrini (surrene, tiroide, pancreas) e non endocrini. Si ritiene che la sindrome si sviluppi in pazienti geneticamente predisposti con diversi pattern genetici del complesso maggiore di istocompatibilità MHC II. La SPA2 è una malattia rara, avendo una frequenza di una persona affetta ogni 7000-8000 abitanti, prevale nel sesso femminile e compare a un'età media di circa 35 anni. L'iposurrenalismo è caratterizzato da sintomi tipici (astenia, ipotensione ortostatica, calo ponderale, artromialgie, nausea, anoressia, iperpigmentazione cutanea), tuttavia non facili da interpretare, data la scarsa conoscenza della malattia. Nei casi non diagnosticati in tempo utile i sintomi possono peggiorare in rapporto a eventi stressanti che possono far precipitare $i$ pazienti in una crisi addisoniana che può essere potenzialmente fatale. Iposodiemia, iperpotassiemia, iperazotemia, ipercalcemia associati ad aumentati livelli plasmatici di ACTH, renina, e bassi livelli di cortisolo, e alterati indici di epatolisi sono riscontri laboratoristici relativamente tardivi, così come possono esserlo i segni clinici di disidratazione. La storia naturale della malattia si manifesta attraverso varie fasi progressive: a) dapprima con presenza di autoanticorpi anti-surrene presenti anni prima all'esordio clinico, b) poi con un aumento della renina plasmatica e con la diminuzione dell'aldosterone plasmatico, c) poi con la successiva ridotta risposta del cortisolo allo stimolo con ACTH e.v. e d) infine con iperincrezione di ACTH, calo del cortisolo basale e presenza delle manifestazioni cliniche di iposurrenalismo. Il trattamento si basa sulla sostituzione ormonale degli organi endocrini coinvolti.

Parole chiave: Morbo di Addison, Poliendocrinopatia autoimmune di tipo 2

Dichiarazione di conflitto di interessi: Gli Autori dichiarano di non avere conflitto di interessi. 
Indirizzo degli Autori:

Dr. Marco Lombardi

Nefrologia e Dialisi

Ospedale SM Annunziata e del Mugello

Azienda Sanitaria di Firenze

Via della Resistenza

Borgo S. Lorenzo

50032 Firenze

lombardim@tin.it ase Type 1, 2, and 4. Ann Endocrinol 2001; 62: 193-201.

15. Owen C, Cheetham T. Diagnosis and management of polyendocrinopathy syndromes. Endocrinol Metab Clin North Am 2009; 38: 419-36.

16. Eisenbarth GS, Gottlieb PA. Autoimmune polyendocrine syndromes. N Engl J Med 2004; 350: 2068-79.

17. Betterle $\mathrm{C}$, Lazzarotto F, Presotto F. Autoimmune polyglandular syndrome Type 2: tip of an iceberg? Clin Exp Immunol 2004; 137: 225-33.

\section{Bibliografia}

1. Addison T. 1855 on the constitutional and local effects of disease of the suprarenal capsules. In a collection of the published writing of the late Thomas Addison, M.D., physician to Guy's Hospital London: Sydenham Society 1868. Reprinted in Medical Classics 1937; 2: 244-93.

2. Schmidt MB. Eine biglandulare Erkrankung (Nebennieren und Schilddrüse) bei Morbus Addisonii. Verh Dtsch Ges Pathol 1926; 21: 212-21.

3. Rowntree LG, Snell AM. A clinical study of Addison's disease. Mayo Clinic Monographs. Philadelphia: WB Saunders Co 1931.

4. Gowen WM. Addison's disease with diabetes mellitus. N Engl J Med 1932; 207: 577-9.

5. Anderson JR, Goudie RB, Gray KG, Timbury GC. Autoantibodies in Addison's disease. Lancet 1957; 1: 1123-4.

6. Neufeld M, Blizzard RM. Polyglandular autoimmune diseases. In: Symposium on autoimmune aspects of endocrine disorders. Pinchera A, Doniach D, Fenzi GF, Baschieri L (Eds). Academic Press, New York 1980: 357-65.

7. Betterle C, Presotto F. Autoimmune Polyendocrine Syndromes (APS) or Multiple Autoimmune Syndromes (MAS). in: Walker S, Jara LJ. (Eds.). Handbook of Systemic Autoimmune Diseases 2008; Vol. 9: 135-48.

8. Ahonen P, Myllarniemi S, Sipila I, Perheentupa J. Clinical variation of autoimmune polyendocrinopathy-candidiasis-ectodermal dystrophy (APECED) in a series of 68 patients. N Engl J Med 1990; 322; 1829-36.

9. Michels AW, Gottlieb PA. Autoimmune polyglandular syndromes. Nature Reviews Endocrinology 2010; 6: 270-7.

10. Majeroni BA, Patel P. Autoimmune polyglandular syndrome, type II. Am Fam Physician 2007; 75 (5): 667-70.

11. Graves L 3rd, Klein RM, Walling AD. Addisonian crisis precipitated by thyroxine therapy: a complication of type 2 autoimmune polyglandular syndrome. South Med J 2003; 96: 824-7.

12. Betterle C, Dal Pra C, Mantero F, Zanchetta R. Autoimmune adrenal insuffi cency and autoimmune polyendocrine syndromes: autoantibodies, autoantigenes, and their applicability in diagnosis and disease prediction. Endocr Rev 2002; 23: 327-364.

13. Coco G, Dal Pra C, Presotto F, et al. Estimated risk for developing autoimmune Addison's disease in patients with adrenal cortex autoantibodies. J Clin Endocrinol Metab 2006; 91: 1637-45.

14. Betterle C, Dal Pra C, Greggio NA, et al. Autoimmunity in isolated Addison's disease and in polyglandular autoimmune dise-

\section{TEST DI VERIFICA}

\section{1) La SPA-2 è una malattia rara: qual è la stima della sua frequenza nel nostro Paese?}
a) $1: 100$
b) $1: 8000$
c) $1: 20000$
d) $1: 100000$

2) La sintomatologia dell'iposurrenalismo primitivo in fase non avanzata di una SPA-2 è:

a) Tipica e facilmente riconoscibile

b) Sovrastata da sintomi neurologici

c) Aspecifica e comune a molte altre patologie

d) Non si riscontra nessun sintomo, indipendentemente dalla fase della malattia

\section{3) Quali alterazioni degli esami di laboratorio caratteriz- zano la sindrome?}

a) IperK, ipoNa, modesta ipercalcemia, ipoglicemia, ipocorticosurrenalismo, aumento ACTH

b) Iperuricemia, segni di disidratazione, iperNa, ipoK, iperglicemia, iporeninismo

c) Ipodisprotidemia, iperfosforemia, iperparatiroidismo, anemia sideropenica

d) Nessuna delle precedenti

\section{4) Qual è la migliore arma a disposizione dei medici per porre diagnosi di morbo di Addison oggi?}

a) La valutazione dei dati antropometrici

b) La misurazione della pressione arteriosa

c) La ricerca degli esami ematochimici alterati

d) La conoscenza della sindrome e della sua clinica

Le risposte corrette alle domande sono pubblicate su questo numero del Giornale di Tecniche Nefrologiche \& Dialitiche Vol. 25, no. 1, pag. 52. 